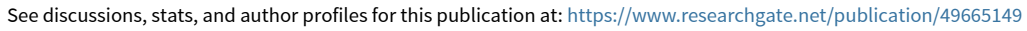

\title{
Force production and spatial arm coordination profile in arm crawl swimming in a fixed position
}

Article in Acta Physiologica Hungarica · December 2010

Dol: 10.1556/APhysiol.97.2010.4.4 Source: PubMed

CITATIONS

7 authors, including:

Karsai Istvan

University of Pécs

42 PUBLICATIONS 1,012 CITATIONS

SEE PROFILE

Hugo Louro

Polytechnic Institute of Santarém

98 PUBLICATIONS 409 CITATIONS

SEE PROFILE

Some of the authors of this publication are also working on these related projects:

Neuromuscular responses in swimming View project

Project

Crossfit for Health and Performance View project
183

Nuno Domingos Garrido

Universidade de Trás-os-Montes e Alto Douro

128 PUBLICATIONS 1,013 CITATIONS

SEE PROFILE

Luís Filipe Moutinho Leitao

Instituto Politécnico de Setúbal

24 PUBLICATIONS 45 CITATIONS

SEE PROFILE 


\title{
Force production and spatial arm coordination profile in arm crawl swimming in a fixed position
}

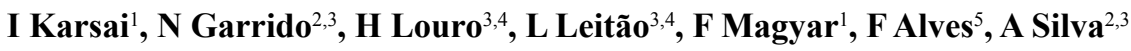 \\ 'Institute of PE and Sport Sciences, University of Pécs, Pécs, Hungary, \\ ${ }^{2}$ Department of PE and Sport, University of Trás-os-Monte and Alto Douro, Vila Real, Portugal, \\ ${ }^{3}$ Research Center for Sport Sciences, Health and Human Development, Vila Real, Portugal, \\ ${ }^{4}$ Sport Sciences School of Rio Maior, Rio Maior, Portugal, \\ ${ }^{5}$ Faculty of Human Movement Kinetics, Technical University of Lisbon, Lisbon, Portugal
}

Received: December 9, 2009

Accepted after revision: May 27, 2010

\begin{abstract}
This study analyzed the relationship between mechanical force production and spatial arm position of the swimming movement for each side of the swimmer. Eight internationally recognized male swimmers performed fix positioned arm only swimming with a dynamometer synchronized with underwater cameras. The upper arm positions ( $\alpha$ in side, $\beta$ in frontal view) and the elbow angles ( $\gamma$ in 3D) were determined at the moment where the force production reached the peak (Fmax) and the maximal values of rate of force development (RFDmax). RFDmax and $\alpha$ values showed significant differences between the sides $(\mathrm{P}<0.05)$. To show the motion integration structure of the performance, Multiple Regression Analysis (MRA) was employed separately for both sides. For the criterion variable, the impulse of force (ImpF50\%) was calculated. The defined parameters as the mechanical and spatial predictor system were used for the model. The results of the MRA showed that the predictor system yielded the model structure of the variables that explain the criterion variables for $\operatorname{ImpF} 50 \%$ by the dominant $(\mathrm{P}=0.007)$ and by the nondominant side $(\mathrm{P}=0.001)$, respectively. The alternate contribution of the variables to the models can objectively express the performance difference between the two sides of the swimmer.
\end{abstract}

Keywords: fix positioned swimming, force-time, spatial position, side difference, model

Swimming performance depends mostly on the optimization of propulsive force production $(2,7,10,14,15)$. Description of the human swimming movement is difficult while the direct force measurement during free swimming is impossible, and the mechanism of the interaction between the hand- and arm complex and water molecules are not fully understood $(3,8,18)$. Several experimental and analytical investigations tried to determine the optimal movement structure of the crawl stroke $(1,5,13)$, but the diversity in anthropometrical characteristics and the complexity of the movement hindered the definition of the general model and find to the relevant parameters for the diagnosis of performance in crawl swimming.

It is reasonable to measure pulling force by tethered swimming methods fixing the swimmers to the edge of the pool or in a stable position that allows them to move their arms freely. Alley (2) and Counsilman (7) measured force developed in crawl arm stroke as a function of velocity using the partially tethered method. They revealed the basic force patterns for tethered crawl swimming. Yeater et al. (21) investigated the tether force for the

\footnotetext{
Corresponding author: Dr. István Karsai

Institute of Physical Education and Sport Science

Faculty of Natural Science, University of Pécs

P.O. Box 266, H-7624 Pécs, Hungary

Phone: +36-72 501-519; Fax: +36-72 501-519

E-mail: karsai@gamma.ttk.pte.hu
} 
crawl, back, and breast strokes and found that the two sides of the swimmer show differences regarding the cross cyclical movement observable by the force time curve.

Previously, the researchers focused mainly on the value of a given peak force (Fmax) or on the average of some peak values (avgFmax ) on the force time curve, and used the mean force value of the time interval (Fmean ). Moreover they tried to find a relationship between sprint swimming velocity (Vmax) and the defined variables. Other researchers developed mathematical models to find the connection between tethered force and force computed by the models. It was established that the models with some modifications, namely taking into account the overlapping effect and adjusting the hydrodynamic coefficients, are valid tools to detect the swimmers actual performance capacity $(1,12)$. Another method to estimate force production and analyze swimming performance is to record their movement using underwater cameras $(4,15)$. The limitation of the method was that the unsteady effects were not taking into account $(9,14,19,20)$. Seifert et al. (16) investigated the influence of the arm coordination asymmetry in crawl swimming using the index of coordination (IdC) developed by Chollet et al. (6). They found that elite swimmers tended to use the overlapping variation of the basic technique in addition there were differences between the values of IdC between the two sides. The effects depend mainly on the motor laterality and the breathing technique utilized. Based on these findings, there is a need to investigate the movement of both arms separately to have a deeper understanding of the technique and the role of the inter-limb coordination during crawl swimming.

In addition to the widely used parameters; Fmax and Fmean of the force time curve to examine tethered force production, there are more specific forms of strength expressions, which can give further information about the quality of movements and their effects in the water. The avgFmax expresses the maximum strength of the involved muscles, and the Fmean the average of the force exertion during a series of cyclic movements, independent from the side differences and overlapping effects. The rate of the force development curve and its maximum value (RFD) expresses the explosive force production of an athlete (17). Evaluating these parameters separately in each stroke they can be used for determining the specific strength. Furthermore the investigation of the spatial positions of the arms at the above-mentioned points of the force time curve can provide important information about arm coordination to diagnose swimming performance. The impulse of force in the essential part of the pulling pattern, between the $50 \%$ values of the Fmax at the ascending and descending slopes of the force time curve (ImpF50\%) expresses the ability of the single arm working potential value in a given period and is an indicator of the propelling force produced by the swimmer. This approach enables the investigation of the difference between the dominant and nondominant sides by avoiding the overlapping effect.

Based on this overview, in this work we investigated the fix positioned crawl arm swimming from two different aspects: to compare the performance of both sides using the selected variables; and to define the relationship between the criterion variable (ImpF50\% as an indicator of propelling potential) and the different dimensions of parameters such as spatial arm position and mechanical force characteristics. 


\section{Materials and Methods}

Sample was composed by eight Portuguese National level swimmers (19.8 \pm 0.9 age; $\mathrm{BM}=$ $73.9 \pm 6.0 \mathrm{~kg} ; \mathrm{BH}=1.81 \pm 0.055 \mathrm{~m}$ ). After completing the written consent form, the swimmers performed fix positioned, only arm crawl, swimming tests with a special water resistant dynamometer (TENZI TNF 06, TENZI Kft., Budapest; Hungary) in the push mode $(200 \mathrm{~Hz})$ (Fig. 1). The dynamometer was connected to a PC and the data was recorded by the TENZI 3.0 program (TENZI Kft., Budapest; Hungary). The test was similar to the fully tethered swimming test used by Yeater et al. (21) and Martin et al. (13) with some modifications. The swimmer was connected to the measurement gage using a helmet instead of wearing a harness around the hip. Previous tests confirmed that the difference between the pull and push mode of the fix positioned swimming was less than $3.5 \%$, and there is no complaints in the neck region during or after performing the push mode test. While the test was being carried out four underwater synchronized cameras $(50 \mathrm{~Hz})$ recorded the motion.

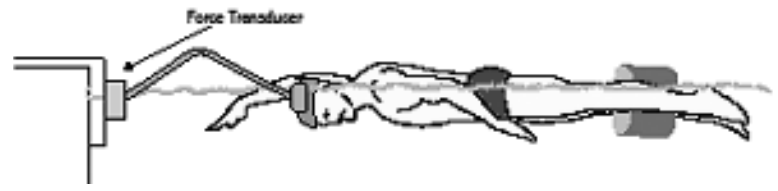

Fig. 1. Fix positioned swimming test, push mode with the water resistant dynamometer

In order to synchronize the kinetic and kinematic data, a control lamp was lit at the moment the force recording started. After a warm up exercise to get familiar with the device, the swimmer started swimming at a low frequency rhythm $(0.41 \mathrm{~Hz})$; the rhythm of a metronome was transmitted using a water resistant earphone. The swimmers were instructed to perform the swimming movement without breathing, one series lasted 20 seconds. The legs were tied and supported during the test and $20 \mathrm{~N}$ forces were applied as a pre-tension force to fix the swimmer in the helmet in order to maintain constant contact with the device. The frequency of strokes was increased gradually $(0.08 \mathrm{~Hz})$ inserting a 1 minute rest between each series. The test was stopped when the swimmer was not able to further increase the frequency of strokes. For the analysis, the averaged results of three whole cycles were used from the series where the highest Fmain was recorded. The kinematic data analysis was performed using the APAS system, according to the spatial position of the center of the shoulder, elbow and wrist joints. To differentiate between the dominant (stronger) and nondominant (weaker) sides, the values of the Fmax of the single strokes were used.

The criterion variable as the time dependent mechanical character of the force production (ImpF50\%) was calculated (Fig. 2) between the 50\% values of the Fmax at the ascending and descending slopes of the force time curve $\left(\operatorname{ImpF} 50 \%=\int_{n=0}^{n} F_{n} * t_{n}\right)$.

To predict the criterion variable, a two-dimensional system was applied where the momentary mechanical and spatial values of the arm movements were determined. For the dominant and nondominant sides (Figs 2 and 3).

i) average of Fmax and the RFDmax of the three whole cycles chosen for the analysis

ii) the momentary spatial positions as a characteristic of arm coordination during stroking maneuvers $\alpha$ (side view), $\beta$ (frontal view) and $\gamma$ (3D) (Fig. 3)

After performing the descriptive statistics to determine the side differences the Wilcoxon Signed Rank test and the Paired Sample $t$-test were used for distribution of the data. The Multiple Regression Analysis (MRA) was used to establish the relationship between the criterion variable and the predictor system for both sides. The analysis was performed using the SPSS v13 for Windows statistical packages. 


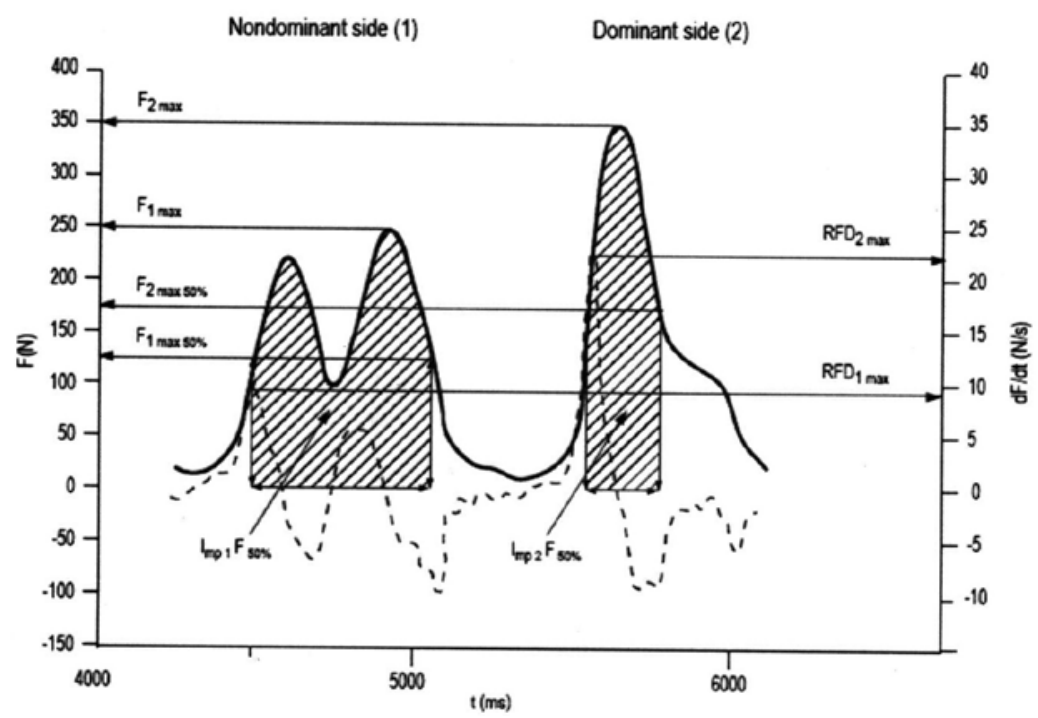

Fig. 2. Force time curve (solid line) and the first derivative of the function (dashed line) to assess the mechanical characteristics of two consecutive arm strokes measured from a representative swimmer. The peaks represent the maximal force production $\left(\mathrm{F}_{\max }\right)$ and the maximal rate of force development $\left(\mathrm{RFD}_{\max }\right)$ for the dominant (stronger) and nondominant (weaker) sides. The filled areas under the force time curve represent the impulse of force (ImpF50\%) determined according to $50 \%$ of $\mathrm{F}_{\max }$

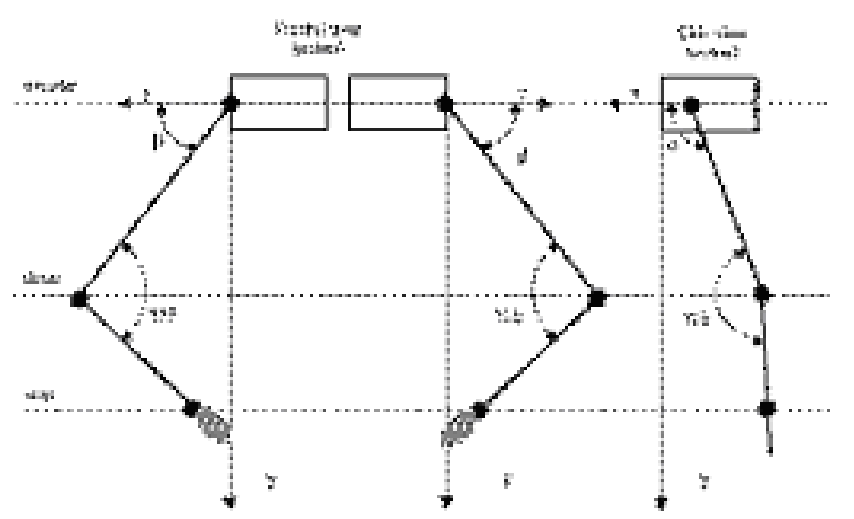

Fig. 3. The spatial positions of the upper arm from a frontal view (yz plane) and side view (xy plane) and the determination of the elbow angle in 3D

\section{Results}

The swimmers $(n=8)$ performed the highest Fmean $=80.42 \mathrm{~N}$ at averaged frequency $(\operatorname{avgFr})$ $0.651 \mathrm{~Hz}$. These results are close to the measured values of the other investigators Akis and Orcan (1) and Yeater et al. (21). The variation coefficients of the Fmean and avgFr; were $12.85 \%$ and $25.82 \%$, respectively. This measurement fits the requirement to use the data as real and valid.

The results obtained from statistical analysis showed significant difference between the dominant and nondominant sides for two mechanical parameters of the measured force production: $\operatorname{Fmax}(\mathrm{z}=2.511, \mathrm{~N}$-Ties $=8, \mathrm{P}=.012$, one-tailed $)$ and $\mathrm{RFDmax}(\mathrm{z}=2.028, \mathrm{~N}$-Ties $=8$, $\mathrm{P}=0.043)$. Furthermore, for one of the spatial arm coordination characters: $\alpha \mathrm{Fmax}(\mathrm{z}=0.224$, $\mathrm{N}-$ Ties $=8, \mathrm{P}=0.025$ ) (Tables I and II). 
The results of ANOVA regression indicates that the system of two-dimensional predictor variables fits the model of predictors that describe the criterion variables statistically with high level of significance for the dominant $(\mathrm{F}=148.803, \mathrm{df}=5.2, \mathrm{P}=0.007)$ and nondominant sides $(\mathrm{F}=198.463, \mathrm{df}=4.3, \mathrm{P}=0.001)$. The model determines the criterion variable by the dominant side $99.7 \%$ and the nondominant side $99.6 \%$ of common variance ( $\mathrm{R}$ square $=$ 0.997 and 0.996). Results of the MRA reveal (Table IV) a difference between the performance structures of the two sides, and describe the different predictor variables that contribute to the models (Tables III and IV).

Table I. Description of the general variables of the fixed position only arm crawl swimming test

\begin{tabular}{|l|c|c|c|c|c|}
\hline & Mean & Min & Max & SD & cV (\%) \\
\hline $\operatorname{avgFr}(\mathrm{Hz})$ & 0.65 & 0.51 & 0.71 & 0.08 & 12.85 \\
\hline Fmean $(\mathrm{N})$ & 80.42 & 51.80 & 103.37 & 20.77 & 25.82 \\
\hline
\end{tabular}

Table II. Descriptive statistics and results of the Wilcoxon Signed Rank test / Paired Samples test for the specified criterion and predictor variables

\begin{tabular}{|c|c|c|c|c|c|}
\hline & \multicolumn{2}{|c|}{ Dominant side } & \multicolumn{2}{|c|}{ Nondominant side } & \multirow[t]{2}{*}{$\mathbf{P}$} \\
\hline & Mean & SD & Mean & SD & \\
\hline ImpF50\% (Ns) & 54.67 & 10.42 & 57.24 & 17.11 & NS \\
\hline Fmax $(\mathrm{N})$ & 275.10 & 61.42 & 212.51 & 43.10 & 0.012 \\
\hline RFDmax (N/s) & 20.92 & 15.82 & 10.18 & 4.84 & 0.043 \\
\hline$\alpha$ Fmax (deg) & 88.53 & 25.89 & 114.88 & 24.17 & 0.025 \\
\hline$\beta$ Fmax (deg) & 49.31 & 24.17 & 55.69 & 15.03 & NS \\
\hline$\gamma$ Fmax (deg) & 117.04 & 9.37 & 110.18 & 21.82 & NS \\
\hline$\alpha$ RFDmax (deg) & 61.99 & 26.31 & 84.17 & 24.28 & NS \\
\hline$\beta$ RFDmax (deg) & 52.35 & 26.99 & 69.05 & 15.10 & NS \\
\hline$\gamma$ RFDmax (deg) & 133.71 & 8.76 & 129.71 & 17.21 & NS \\
\hline
\end{tabular}

$\mathrm{NS}=$ nonsignificant

Table III. Results of ANOVA of regression of the models for the dominant and nondominant sides

\begin{tabular}{|c|c|c|c|c|c|}
\hline & $\begin{array}{l}\text { Sum of } \\
\text { squares }\end{array}$ & df & $\begin{array}{l}\text { Mean } \\
\text { square }\end{array}$ & F ratio & $\mathbf{P}$ \\
\hline Model for the dominant side & 1383.36 & 5 & 276.673 & 148.80 & 0.007 \\
\hline Residual & 3.719 & 2 & 1.859 & & \\
\hline Total (Corr.) & 1387.08 & 7 & & & \\
\hline Model for the nondominant side & 1445.52 & 4 & 361.380 & 198.46 & 0.001 \\
\hline Residual & 3 & 1.82 & & & \\
\hline Total (Corr.) & 1450.98 & 7 & & & \\
\hline
\end{tabular}


Table IV. The parameters of the models (Multiple Regression Analysis - Enter method)

\begin{tabular}{|l|c|c|c|c|c|}
\hline \multirow{2}{*}{} & \multicolumn{2}{|c|}{ Unstandardized coeff. } & $\begin{array}{c}\text { Standar- } \\
\text { dized coeff. }\end{array}$ & \multirow{2}{*}{ t } & \\
\cline { 2 - 5 } & B & Std. err & Beta & & \\
\hline Model for the dominant side & & & & & \\
\hline (Constant) & 247.394 & 9.738 & & 25.404 & 0.002 \\
\hline Fmax & -0.220 & 0.19 & -1.042 & -11.770 & 0.007 \\
\hline$\alpha$ Fmax & 0.381 & 0.037 & 0.665 & 10.441 & 0.009 \\
\hline$\beta$ Fmax & 0.883 & 0.038 & 1.465 & 23.467 & 0.002 \\
\hline$\gamma$ Fmax & -2.045 & 0.092 & -1.625 & -22.310 & 0.002 \\
\hline RFDmax & 2.00 & 0.170 & 1.215 & 11.773 & 0.007 \\
\hline Model for the Nondominant side & & & & & \\
\hline (Constant) & -157.625 & 15.867 & & -9.928 & 0.002 \\
\hline$\alpha$ Fmax & 0.311 & 0.047 & 0.632 & 6.629 & 0.007 \\
\hline RFDmax & 1.883 & 0.255 & 0.699 & 7.390 & 0.005 \\
\hline$\gamma$ RFDmax & 1.085 & 0.052 & 1.298 & 20.750 & 0.001 \\
\hline$\beta$ Fmax & 0.329 & 0.070 & 0.375 & 4.706 & 0.018 \\
\hline
\end{tabular}

\section{Discussion}

The results obtained provided two groups of findings related to the specific aims of the present study. On one hand, in some cases significant differences exist among the examined variables between the two sides, on the other hand the ANOVA regression model provided a statistically high level of significance for the criterion variables for both sides and the structures of the models differ from each other.

\section{Asymmetry between the sides}

The asymmetric pulling patterns between the two arms during tethered swimming were observed by Yeater et al. (21) and Rushall et al. (14). They concluded that there were differences between the peak forces (Fmax) of the two sides. Seifert et al. (16) observed changes between the dominant and nondominant sides using the index of coordination (IdC) method. Our results, utilizing another point of view, underpin the previous findings. The Fmax and RFDmax differ as mechanical characteristics vary significantly between the sides. The notably different results of $\alpha \mathrm{Fmax}$ as the spatial coordination parameter indicates, that the dominant hand reaches Fmax at the mechanically optimalized position near 90 degrees in the xy plane of the shoulder position to the horizontal plane as was demonstrated by Lauder and Dabnichki (11). The nondominant side produced its RFDmax close to the mechanically optimal position regarding the $\alpha$ value and reaches its Fmax with $26.7 \%$ lower values than the dominant side and 24.4 degrees behind the vertical projection of the shoulder joint center. The mean elbow angle at the examined points on either side showed almost constant values, the means ranged between 110.18 and 133.71 degrees in our study. The favorable elbow flexion angle between free swimming and fix positioned swimming should differ, but according to our study, the elbow joint position remains constant with both sides. The upper arm position in the yz plane did not show considerable difference between the two sides. 


\section{Comparison of the MRA results}

The model includes parameters that predict the impulse force (ImpF50\%) in different ways which is representative of the working potential of the moving arms in the fix positioned swimming test. Despite the finding that the ImpF50\% values are almost the same in both cases (diff. $4.49 \%$ ) for the dominant and nondominant side, the model structure differs by the $t$ value of the constants $(25.4$ and -9.92$)$. While the results of the models with the reliability rate of $99.6 \%$ and $99.6 \%$ show dependence, the contribution of the different parameters of the structures, we can assume that the difference exists because of the way the motion is organized. The model for the dominant side consists of five parameters, four of them related to the maximal force achievement of one side (Fmax, $\alpha$ Fmax, $\beta$ Fmax, $\gamma$ Fmax) and RFDmax. The model shows that the motion is structured to produce a higher force during the stroke. The force peak (Fmax) is reached earlier than the peak by the nondominant side and the time duration is shorter during the essential part of the stroke. The model for the nondominant side consists of two parameters associated with the RFDmax and two components associated with Fmax. The lower beta values and the selected parameters for the model, provide evidence that none of the pre-determined characters play a leading role in the organization to build the structure. We assume that during a longer period of moderate force production, the required force impulse is produced without attempting to reach a high value of Fmax or RFDmax. From these findings arises the question whether it is the complicated hydro dynamical circumstances and a lack of the neuromuscular system liable for the asymmetry, or rather, when the ImpF50\% does not differ considerably (diff. 4.49\%) an existent compensatory system which regulates the reconsiliation between the sides is shown.

In conclusion, the results of this study suggest that under the investigated circumstances, in addition to the Fmax, the side difference is present in two other parameters in RFDmax and $\alpha$ Fmax. Furthermore, by applying the used method to evaluate the kinetic and kinematic characteristics of the cross-cyclical crawl swimming movement together provides meaningful information from the performance structure. The trainers and athletes are able to handle the problems which may arise from the difference in sides of the swimmer.

\section{REFERENCES}

1. Akis T, Orcan Y: Experimental and analytical investigation of the mechanics of crawl stroke swimming. Mech. Res. Commun. 31, 243-261 (2004)

2. Alley LE: An analysis of water resistance and propulsion in swimming the crawl stroke. Res. Q. Exerc. Sport. 23, 253-270 (1952)

3. Berger MA, Hollander AP, de Groot G: Technique and energy losses in front crawl swimming. Med. Sci. Sports Exerc. 29, 1491-1498 (1997)

4. Berger MA, Hollande, AP, de Groot G: Determining propulsive force in front crawl swimming: a comparison of two methods. J. Sports Sci. 17, 97-105 (1999)

5. Bixler B, Schloder M: Computational fluid dynamics. An analytical tool for the 21 st century swimming Scientist. J. Swim. Res. 11, 4-22 (1996)

6. Chollet D, Chalies S, Chatard JC: A new index of coordination for the crawl: description and usefulness. Int. J. Sports Med. 21, 54-59 (2000)

7. Counsilman J: Forces in swimming two types of crawl strokes. Res. Q. Exerc. Sport. 26, 127-139 (1955)

8. Deschodt VJ, Arsac LM, Rouard AH: Relative contribution of arms and legs in humans to propulsion in 25-m sprint frontcrawl swimming. Eur. J. Appl. Physiol. 80, 192-199 (1999)

9. Dickinson MH: Unsteady mechanisms of force generation in aquatic and aerial locomotion. Am. Zoologist. 36, 537-554 (1996) 
10. Haffner M, Cappaert M (1999): Underwater analysis of the freestyle stroke from three different points in the stroke cycle. In: Biomechanics and Medicine in Swimming VIII, Eds. Keskinen K, Komi P, Hollander AP, Gummerus Printing, Jyvaskyla, pp. 153-157

11. Lauder MA, Dabnichki P: Estimating propulsive forces sink or swim? J. Biomech. 38, 1984-1990 (2005)

12. Magel JR: Propelling force measured during tethered swimming in the four competitive styles. Res. Q. Exerc. Sport. 41, 68-74 (1970)

13. Martin RB, Yeater RA, White MK: A simple analytical model for the crawl stroke. J. Biomech. 14, 539-548 (1981)

14. Rushall BS, Springings EJ, Holt LE, Cappaert JM: A re-evaluation of forces in swimming. J. Swim. Res. 10, 6-30 (1994)

15. Schleihauf RE (1979): A hydrodynamic analysis of swimming propulsion. In: Swimming III. Eds. Terauds J, Clarys JP, International Series on Sport Sciences, University Park Press, Baltimore, pp. 70-109

16. Seifert L, Chollet D, Allard P: Arm coordination symmetry and breathing effect in front crawl. Hum. Mov. Sci. 24, 234-256 (2005)

17. Siff MC, Verkhoshansky YV (1998): Supertraining. University of the Witwatersrand, Johannesburg

18. Toussaint HM, Beek PJ: Biomechanics of competitive front crawl swimming. Sport Med. 13, 8-24 (1992)

19. Toussaint HM (2000): An alternative fluid dynamic explanation for propulsion in front crawl swimming. In: Proceedings of the XVIII International Symposium on Biomechanics in Sports. Eds. Sanders R, Hong Y, Chinese University of Hong Kong, Hong Kong, pp. 96-103

20. Toussaint HM, Van De Berg C, Beek WJ: "Pumped-up propulsion” during front crawl swimming. Med. Sci. Sports Exer. 34, 314-319 (2002)

21. Yeater RA, Martin RB, White MK, Gilson KH: Tethered swimming forces in crawl, breast and back strokes and their relationship to competitive performance. J. Biomech. 14, 527-537 (1981) 